\title{
Response by Caplan et al
}

\author{
Arthur L Caplan ${ }^{1}$, Carolyn Plunkett ${ }^{1,2}$, Brendan Parent ${ }^{1} \&$ Michael Shen $^{3}$
}

Reply to: $\mathbf{M}-\mathbf{L}$ Wong (in this issue)

W e would like to thank Dr. Wong for the response to our article "No time to waste-the ethical challenges created by CRISPR". Dr. Wong's letter addresses a question about the relationship between biology and society: that "prevailing papers" on CRISPR-Cas9 rarely emphasize understanding the microbiological processes that make CRISPR-Cas9 an effective defensive mechanism. Instead, scientists and society are more interested in figuring out how CRISPR-Cas9 can be used to fix society's problems - creating "better" organisms or eradicating "bad" bugs. Dr. Wong's maintains that those researching CRISPR-Cas9 should be more interested in understanding and teaching society about, quoting Carl Woese, "its world and itself".

But, why can't scientists do both? To insist that one pursuit is better or nobler than another presents a false dichotomy. Biologists in Carl Woese's view can only describe nature or alter it. In reality, they can, and should, do both—what journals publish is another matter.

It is true that a focus on applications tends to swamp fundamental understanding in the eyes of a public and media eager to hear news of cures. But, as we argue in our study, the need to base application on a sound understanding of the fundamental science is a powerful moral imperative. The noise from some of the hype surrounding CRISPR should not obscure that ethical requirement. 